\title{
The power of Mu Xiang to treat inflammatory bowel disease
}

\section{Commentary}

Bloating and gas pain is something that everyone has had to deal with at one point or another; however, that's usually reserved for holiday dinners and other large gatherings. Too much turkey dinner on Thanksgiving can leave the most robust stomach and individual incapacitated on the floor for a good portion of the evening cursing themselves for indulging in that third helping of dinner and pie.

For the person dealing with an IBD like Crohn's or Ulcerative Colitis this can be an every meal occurrence. In the fall of 2011 was diagnosed with Crohn's disease after losing 30 pounds in a month. With the help of Western Medicine and Chinese Medicine I have gained back the 30 pounds. I have been fortunate enough to have learned acupuncture and Chinese Medicine and have been able to utilize the vast knowledge of the past centuries in order to keep myself out of a "flare" for over two years. I would like to take this time to shed some light on an herb that has played an important role in my life and in my patients with digestive issues as well.

The ancient Taoists viewed the processes within our bodies similar to the processes of nature around us. From this metaphor came the five elements: Fire, Earth, Metal, Water and Wood and the six "pathogenic" factors: Wind, Cold, Heat, Dampness, Dryness, and Summer Heat. Each of the five elements corresponds with a paired organ system and those organs can be damaged by the six pathogenic factors. For example, the Lungs can be damaged by Wind resulting in runny nose and sneezing as in allergies. We know that allergies can come from pollen, dander and dust but wind is the transporter of the allergens. Another example would be a person out in the hot sun without enough water coming down with heat stroke. Too much heat has affected the body's natural temperature control and the person gets sick. Now that we have a basic grasp on how the six pathogenic factors can affect our health we can turn our focus on how Chinese Medicine ${ }^{1}$ uses that same thought process to heal the body.

We know that there are five basic tastes or flavors to foods: sweet, salty, sour, acrid/spicy and bland. What we tend to not think of is the "temperature" of a particular food has a great role in affecting our health and well being. This "temperature" scale ranges in various degrees from hot to cold. With a little bit of thought this can be understood very easily. For example a bite into a chili pepper will cause the mouth to get hot and burn.

That's all the way on the "hot" side of the spectrum. The "cold" foods are harder for us to distinguish in general, but a basic way to understand this is to look at types of foods we tend to gravitate towards during the seasons. Let's look at a watermelon. During the summer months when it's 100 degrees out, a bite into a room temperature watermelon is still so juicy, sweet, refreshing and cooling to the system. In essence, foods grown in the summer months tend to be cool or cold in temperature and foods grown in the winter months tend to be more warming or hot in temperature. When a person eats and lives in accordance with the seasons their body will function at an optimal level and disease will not manifest. This is because the $q i$ and Blood are circulating through the entire body all the way down to the cellular level. $Q i$ and Blood refer to the blood, nutrients, oxygen and other "life giving" processes that are happening within our bodies at
Volume I Issue 2 - 2015

\author{
Drew Nystrom \\ Precision Point Wellness, USA
}

Correspondence: Drew Nystrom, Licensed Acupuncturist, Precision Point Wellness, 14340 Bolsa Chica Rd, Suite G Westminster, CA 92683, USA, Tel 7147098030, Email drew@precisionpointwellness.com

Received: May 12, 2015 | Published: May 25, 2015

every moment of every day of our lives. When the blood, nutrients, and oxygen are in high quality, being absorbed and all the processes are functioning at optimal levels we will stay healthy, youthful, vibrant and disease free. It isn't until a process fails or we starve our cells of oxygen and nutrients that the sickness starts. Like everything our bodies have a threshold or breaking point and it isn't until the "disease" goes beyond the breaking point do we start to notice symptoms.

Now let's take a look at a particular herb called "Mu Xiang". $M u$ Xiang, which literally translates to "wood fragrance", is also known as Radix Auclandiae Lappe (Latin) or Costus root (English) and is in the "Regulate $Q i$ " category in the Chinese Herbal Pharmacopoeia. ${ }^{2}$ "Regulate Qi" can be translated as "to keep processes/functions moving properly". $M u$ Xiang's function is to regulate qi movement and alleviate pain associated with the digestive tract. Three indications to use this herb would be:

I. Qi stagnation in the epi-gastrium and abdomen, which causes distention.

II. This can be seen as a blockage of food within the intestines and/or peristalsis (the smooth muscle contraction, which pushes the food along) isn't functioning properly.

III. Qi deficiency leading to loss of appetite and oppression fullness in the epi-gastrium and abdomen along with nausea.

IV. This can be seen as a lack of muscle tone in the digestive tract and/or decreased gastric juices so food isn't being broken down appropriately.

V. Painful diarrhea accompanied by abdominal pain and tenesmus (a feeling of an incomplete bowel movement)

VI. Not only can $M u$ Xiang increase muscle tone it can also decrease muscle spasm causing cramps, diarrhea and swelling of the tissues.

Mu Xiang treats the symptoms and it also, mildly, treats the source/ root of the problem by strengthening the spleen and stomach (i.e. digestion). In a study done on mice in 2005 Radix Aucklandiae [3] had "surprising inhibition activity on hydrochloric acid-ethanol type acute gastric ulcer $(\mathrm{P}<0.01)$ [3]. It also could obviously reduce the index of gastric ulcer induced by reserpine and chronic gastric ulcer induced 
by acetic acid $(\mathrm{P}<0.05-0.01)$." With some scientific evidence stating that Radix Aucklandiae (Mu Xiang) [3] helps reduce gastric ulcers let us take a look at some basic formulary examples an herbalist might use for a patient with IBD: Xiang Sha Liu Jun Zi Tang "Six Gentlemen Decoction with Aucklandiae and Amomum" is used for "Spleen $Q i$ deficiency with Phlegm-Dampness and pain" and "Stomach Cold". Some of the symptoms this formula treats are nausea, vomiting, emaciation, lack of appetite with full feeling after eating very little, acid regurgitation, epigastric and abdominal distention and pain, loose stools and diarrhea, fatigue. The pulse will be deep and weak The tongue will show a thin white coat and a pale flabby body with possible teeth marks.

Zhen Ren Yang Zang Tang “True man's decoction to nourish the organs" treats a Spleen and Kidney Yang deficiency by warming the middle/abdomen, strengthening the spleen and stomach, astringing the intestines to stop diarrhea. Manifestations of this pattern present as chronic diarrhea/dysentery with possible blood and/or mucus, fecal incontinence, tenesmus, abdominal pain that's better with warmth, reduced appetite, poor appetite, and lower back pain. The pulse will be thin and slow and the tongue will be pale with a white coat. ${ }^{4}$

Shao Yao Tang "Peony Decoction" treats a Large Intestine DampHeat with $Q i$ and Blood stagnation causing bleeding by unblocking the $q i$ and blood, clearing heat, draining dampness, resolving toxicity and pain. Some of the symptoms would include: abdominal pain, difficulty defecating, blood and pus in stool, burning sensation around the anus, fever and no thirst. The pulse is slippery and rapid and the tongue will be greasy slightly yellow coat. Every herb is categorized by taste and temperature and which organs/ channels it goes to. $M u$ Xiang is acrid, bitter and warm and because of how this herb works we know, in the Chinese Pharmacopoeia, ${ }^{2}$ that the "channels" or organs
Mu Xiang enters is the Gallbladder, Large Intestine/Colon, Spleen and the Stomach. Due to the acrid, bitter and warm properties Mu Xiang is contraindicated for use with patients who have depleted fluids and yin. In the event of a patient who presents with a yin deficiency or Blood Heat diagnosis, herbs like Sheng Di Huang (Rehmanniae Radix), Xuan Shen (Scrphulariae Radix), Zi Cao (Lithospermi Radix), Mu Dan Pi (Moutan Cortex) and Chi Shao (Paeoniae Radix Rubra) should be used in conjunction.

\section{Acknowledgments}

None.

\section{Conflict of interest}

The authors declare that there is no conflict of interest.

\section{Funding}

None.

\section{References}

1. Dan B, Andrew G. Chinese Herbal Medicine: Materia Medica (Revised edn). Seattle, USA: Eastland Press; 1993. pp. 237-238.

2. Zhang Ji. Herbal Pharmacopoeia 3. 2007.

3. Han J, Lin H, Zhong Z, et al. Study of the effect of exceed critical extracts from Radix Aucklandiae on experimental gastric ulcer Model. Zheng Yao Cai. 2005;28(11):1017-1019.

4. Dan B, Andrew G. Chinese Herbal Medicine: Formulas \& Strategies, $2^{\text {nd }}$ edn. Seattle, USA: Eastland Press; 1990. pp. 1019. 